Received 2012 May 31; ACCepted 2012 August 17; Published 2012 September 26

Preprint typeset using $\mathrm{L}^{\mathrm{A}} \mathrm{T} \mathrm{E}$ X style emulateapj v. 5/2/11

\title{
A RADIAL VELOCITY STUDY OF COMPOSITE-SPECTRA HOT SUBDWARF STARS WITH THE HOBBY-EBERLY TELESCOPE*
}

\author{
Brad N. Barlow ${ }^{1 \dagger}$, Richard A. Wade ${ }^{1}$, Sandra E. Liss ${ }^{1}$, Roy H. Østensen ${ }^{2}$, \& Hans Van Winckel ${ }^{2}$ \\ ${ }^{1}$ Department of Astronomy \& Astrophysics, The Pennsylvania State University, 525 Davey Lab, University Park, PA 16802 \\ ${ }^{2}$ Instituut voor Sterrenkunde, K.U. Leuven, B-3001 Leuven, Belgium \\ Received 2012 May 31; accepted 2012 August 17; published 2012 September 26
}

\section{ABSTRACT}

Many hot subdwarf stars show composite spectral energy distributions indicative of cool main sequence companions. Binary population synthesis (BPS) models demonstrate such systems can be formed via Roche lobe overflow or common envelope evolution but disagree on whether the resulting orbital periods will be long (years) or short (days). Few studies have been carried out to assess the orbital parameters of these spectroscopic composite binaries; current observations suggest the periods are long. To help address this problem, we selected fifteen moderately-bright (V 13) hot subdwarfs with F-K dwarf companions and monitored their radial velocities (RVs) from January 2005 to July 2008 using the bench-mounted Medium Resolution Spectrograph on the Hobby-Eberly Telescope (HET). Here we describe the details of our observing, reduction, and analysis techniques and present preliminary results for all targets. By combining the HET data with recent observations from the Mercator telescope, we are able to calculate precise orbital solutions for three systems using more than 6 years of observations. We also present an up-to-date period histogram for all known hot subdwarf binaries, which suggests those with $\mathrm{F}-\mathrm{K}$ main sequence companions tend to have orbital periods on the order of several years. Such long periods challenge the predictions of conventional BPS models, although a larger sample is needed for a thorough assessment of the models' predictive success. Lastly, one of our targets has an eccentric orbit, implying some composite-spectrum systems might have formerly been hierarchical triple systems, in which the inner binary merged to create the hot subdwarf.

Subject headings: binaries: spectroscopic — ephemerides — subdwarfs — techniques: radial velocities

\section{INTRODUCTION}

Binarity plays an essential role in the story of the hot subdwarf B ( $\mathrm{sdB})$ stars, one of the most enigmatic stages of stellar evolution. The sdBs dominate surveys of faint blue objects and are found in almost all Galactic stellar populations. They are the field counterparts of the Extreme Horizontal Branch stars in globular clusters, and their location in the $\mathrm{H}-\mathrm{R}$ diagram corresponds to stellar models with $\mathrm{He}$-burning cores and extremely thin $\mathrm{H}$ envelopes (Heber 1986). Presumably, the sdB progenitors were stripped of almost all their surface hydrogen while on or near the red giant branch (RGB), igniting helium burning in their cores at nearly the same time. The question is how this can happen, since most Population I stars do not lose their entire envelopes, and remain in the red clump, not far from the RGB, during core $\mathrm{He}$ burning.

Stochastic mass loss from single stars (D'Cruz et al. 1996) or other single-star scenarios to explain this are ad hoc, so most recent effort has concentrated on mass loss resulting from interactions in a close binary star system, following the early exploration by Mengel et al. (1976). Han et al. (2002, 2003), for example, described five channels of binary star evolution that can lead to the formation of hot subdwarfs: the 'first' and 'second'

\footnotetext{
* Based on observations obtained with the Hobby-Eberly Telescope, which is a joint project of the University of Texas at Austin, the Pennsylvania State University, Stanford University, Ludwig-Maximilians-Universität München, and Georg-AugustUniversität Göttingen.

† bbarlow@psu.edu
}

stable Roche-lobe overflow (RLOF) scenarios, the 'first' and 'second' common envelope (CE) channels, and the merger of two He-core white dwarfs (WDs). Their CE scenarios produce close binaries with orbital periods from hours to days; the sdB companions are generally latetype $(\mathrm{G} / \mathrm{K} / \mathrm{M})$ main sequence stars or white dwarfs. In contrast, their stable RLOF channels lead to systems with early-type (B/A/F) main sequence or late-type giant companions and long orbital periods $(P \sim$ few $\times 100$ d). An alternative scenario employing the $\gamma$-formalism has been put forth by Nelemans et al. (2000, 2001) and Nelemans (2010), whose CE channels can produce systems with main sequence companions and periods on the order of years.

To date, orbital parameters have been measured for approximately $150 \mathrm{sdB}$ binary systems; the overwhelming majority of these are close binaries with $P<$ 15 d (see Table A.1 of Geier et al. 2011 for a recent summary). The companions are mostly WDs and M dwarfs, essentially invisible in the glare of the sdB. With a suitable choice of tuning parameters, theoretical models can reproduce the observed distribution of sdB masses and orbital periods in these systems reasonably well (Maxted et al. 2001; Copperwheat et al. 2011). The recent binary population synthesis (BPS) study by Clausen et al. (2012), however, shows that numerous parameter sets can reproduce this observed subpopulation; thus, additional constraints are needed to better tune BPS codes.

Very little attention has been paid to the $\mathrm{sdB}+\mathrm{F} / \mathrm{G} / \mathrm{K}$ binaries, in spite of the nearly equal flux contributions 
TABLE 1

RV MONITORING TARGETS

\begin{tabular}{|c|c|c|c|c|c|c|}
\hline Target & Sp. Type ${ }^{a}$ & $\begin{array}{l}\mathrm{RA} \\
{[\mathrm{J} 2000]} \\
\end{array}$ & $\begin{array}{l}\text { Dec } \\
{[\mathrm{J} 2000]}\end{array}$ & $\mathrm{V}$ & $\begin{array}{l}\text { Alternate } \\
\text { Name }\end{array}$ & Comments \\
\hline PG $0039+049$ & $\mathrm{sdB}+\mathrm{G} 2 \mathrm{~V}$ & $00: 42: 06.1$ & $+05: 09: 24$ & 12.9 & PB 6107 & \\
\hline PG $0110+262$ & $\mathrm{sdB}+\mathrm{G} 0 \mathrm{~V}$ & $01: 13: 14.9$ & $+26: 27: 31$ & 12.9 & & \\
\hline PB 8783 & $\mathrm{sdOV}^{b}+\mathrm{F} 4 \mathrm{~V}$ & $01: 23: 43.2$ & $-05: 05: 45$ & 12.3 & EO Cet & pulsating hot subdwarf ${ }^{5}$ \\
\hline PHL 1079 & $\mathrm{sdB}+\mathrm{G} 7 \mathrm{~V}$ & $01: 38: 27.1$ & $+03: 39: 39$ & 13.4 & & \\
\hline PG $0232+095$ & $\mathrm{sdB}+\mathrm{G} 1 \mathrm{~V}$ & $02: 35: 12.0$ & $+09: 45: 38$ & 12.5 & & \\
\hline PG $1040+234$ & $\mathrm{sdB}+\mathrm{G} 1 \mathrm{~V}$ & $10: 43: 39.3$ & $+23: 09: 07$ & 13.4 & TON 1281 & resolved $^{1}$ \\
\hline PG $1104+243$ & $\mathrm{sdOB}+\mathrm{G} 2 \mathrm{~V}$ & $11: 07: 26.3$ & $+24: 03: 12$ & 11.3 & & MS orbit published ${ }^{4}$ \\
\hline PG $1206+165$ & $\mathrm{sdB}+\mathrm{G} 9 \mathrm{~V}^{c}$ & $12: 09: 16.7$ & $+16: 11: 56$ & 13.8 & PB 3854 & \\
\hline PG $1253+284$ & $\mathrm{sdB}+\mathrm{F} 8 \mathrm{~V}$ & $12: 56: 04.9$ & $+28: 07: 20$ & 12.7 & TON 139 & sdB orbit published ${ }^{3} ;$ resolved $^{1} ;$ presumed triple system $^{1,3}$ \\
\hline PG $1317+123$ & $\mathrm{sdO}+\mathrm{G} 1 \mathrm{~V}$ & $13: 19: 53.6$ & $+12: 03: 59$ & 11.3 & Feige 80 & MS orbit published ${ }^{4}$ \\
\hline PG $1338+611$ & $\mathrm{sdB}+\mathrm{G} 4 \mathrm{~V}$ & $13: 40: 14.7$ & $+60: 52: 48$ & 11.4 & Feige 87 & MS orbit published ${ }^{4}$ \\
\hline PG $1449+653$ & $\mathrm{sdB}+\mathrm{G} 5$ & $14: 50: 36.1$ & $+65: 05: 53$ & 13.6 & & \\
\hline PG $1629+081$ & $\mathrm{sdOB}+\mathrm{K} 5 \mathrm{~V}^{c}$ & $16: 32: 01.4$ & $+07: 59: 40$ & 12.8 & & resolved $^{2}$ \\
\hline PG $1701+359$ & $\mathrm{sdB}+\mathrm{G} 8$ & $17: 03: 21.6$ & $+35: 48: 49$ & 13.2 & & \\
\hline PG $1718+519$ & $\mathrm{sdB}+\mathrm{G} 4 \mathrm{~V}$ & $17: 19: 45.5$ & $+51: 52: 10$ & 13.7 & & resolved $^{1}$ \\
\hline
\end{tabular}

from the two components in such systems. The relative dearth of published orbital parameters implies their periods are quite long. More than a decade ago, Green et al. (2001) hinted that sdB binaries with composite spectra tend to have longer orbital periods. They reported an average around 3-4 years, although no specific systems were named. Since that brief discussion, only two other studies have claimed the detection of orbital periods in composite--spectrum binaries, both of which were published very recently. Østensen \& Van Winckel (2012) (hereafter $\varnothing 12$ ) discuss a survey conducted with the High Efficiency and Resolution Mercator Echelle Spectrograph (HERMES, see Raskin et al. 2011) mounted at the Mercator Telescope on La Palma, Spain and present preliminary results showing orbital periods longer than $\sim$ $500 \mathrm{~d}$ for at least eight binaries. In addition to that work, Deca et al. (2012) report a period of $760 \mathrm{~d}$ for PG 1018-047.

Orbits with such long periods are sufficiently large to have once accommodated an inner binary, so it is possible that these systems were formerly hierarchical triple systems, in which case the subdwarf could have formed from the merger of the inner binary. Clausen \& Wade (2011), for example, recently proposed a scenario in which the merger of a He-core WD and a low-mass main sequence star could eventually form an sdB. If such a binary were in orbit with a dwarf companion, the merger would leave behind an sdB and a main sequence star that had no part in the formation of the sdB (aside from potentially advancing the merger process via the Kozai mechanism). Unlike stable RLOF- and CE-produced systems, which should have nearly circular orbits, no limitations exist on the eccentricities of these binaries other than a requirement that the periastron separation not be too small. Thus, it is imperative to constrain the orbital geometry in addition to the period and radial velocity (RV) amplitudes.

Here we describe a survey carried out with the HobbyEberly Telescope $(\mathrm{HET})$ over $\approx 3.5$ years to measure the line- of-sight accelerations of a sample of sdBs with com- posite spectra. We discuss our target selection in $₫ 2$ and observing strategies in 33 . The details of our analysis techniques for determining velocities and fitting the RV curves are given in 4 . Preliminary results and the current status of our program are presented in \$5. After combining the HET data with recent measurements from $\varnothing 12$, we report precise orbital solutions for three of the targets and discuss them in greater detail. Our initial results confirm early suggestions that the orbital periods of many $\mathrm{sdB}+\mathrm{F} / \mathrm{G} / \mathrm{K}$ systems are long, on the order of several years. We present in $₫ 6$ an updated orbital period histogram for all measured sdB binaries, which shows a possible gap in the period distribution centered near $P=70$ days, and we briefly discuss the puzzle that it presents and possible resolutions. Finally, we summarize our results in $₫ 7$

\section{TARGET SELECTION}

Our criteria for selecting targets were simple: targets were required to be (i) hot subdwarfs with late $\mathrm{F}$, $\mathrm{G}$, or early $\mathrm{K}$ dwarf companions, (ii) relatively bright ( $\mathrm{V} \approx 11-14$ ), and (iii) observable from the HET, situated at McDonald Observatory. F/G/K main sequence companions have similar enough luminosities to hot subdwarfs that these systems show composite spectra at optical and/or infrared wavelengths. The cool companions' rich spectra provide a plethora of lines for precise RV measurement and match the solar spectrum (G2 V) well enough that twilight sky spectra obtained nightly can be used as high signal-to-noise $(\mathrm{S} / \mathrm{N})$ ratio RV templates. Informed by previous observations from Green et al. (2001), we selected fifteen binaries meeting the above criteria, as presented in Table 11. All targets were recognized as potential hot subdwarf systems in the Palomar-Green Survey (Green et al. 1986), except for PHL 1079 (Haro \& Luvten|1962; Kilkenny 1984) and PB 8783 (Berger \& Fringant 1984). Rough spectral types for the cool companions have been published for most of the targets, but since these come from different sources and sometimes differ significantly, we classified them our- 
selves as described in 4.1

Several of the targets have been observed in greater detail already. Heber et al. (2002) and Østensen et al. $(2005)$, for instance, found that four of the systems are resolved binaries with separations up to $2^{\prime \prime}$. Of these, PG $1253+284$ has been recognized as a potential triplestar system due to the detection of a rapid acceleration of the sdB star that is incompatible with the separation implied by the $0^{\prime \prime} .32$ angular distance between the two observed components. PB 8783 exhibits stellar pulsations (Koen et al. 1997) with surface velocities that complicate the characterization of any orbital accelerations around its F-type companion. Most notably, three of our targets (PG 1104+243, PG 1317+123, and PG 1338+061) were recently observed in an RV monitoring program similar to ours by $\varnothing 12$, who report circular orbit solutions from measurements of the cool companion lines. In $\$ 5$, we combine the HET data with their more recent results to gain a total observing baseline of $\sim 6 \mathrm{yr}$ for these binary systems.

\section{HET OBSERVATIONS AND REDUCTIONS}

Over a three-year period from 2005 to 2008, we monitored all survey targets spectroscopically with the bench-mounted Medium Resolution Spectrograph (MRS; Ramsey et al. 1998) on the 9.2-m HET. The HET operates in a queue-scheduled mode. Follow-up observations of some of our targets are currently being carried out with the High Resolution Spectrograph (HRS) on HET and will be discussed in a future paper. We used the "blue" $1.5^{\prime \prime}$ optical fiber pair, the $220 \mathrm{~mm}^{-1}$ crossdisperser grating, and $2 \times 2$ on-chip binning to achieve an average resolution of $R=10,000$ over the $4400-6200$ $\AA$ spectral range. This configuration yields an average dispersion of $\simeq 10 \mathrm{~km} \mathrm{~s}^{-1}$ per binned pixel and samples $\sim 3.2$ pixels per resolution element. The pair of fibers, which are separated by $10^{\prime \prime}$, allowed us to monitor the sky flux and target flux simultaneously. Exposure times ranged from 180 to $1800 \mathrm{~s}$ depending on stellar magnitude, giving us a typical $\mathrm{S} / \mathrm{N}$ ratio of 80 per resolution element.

Each object spectrum was preceded and/or followed by a pair of ThAr comparison lamp spectra (with different exposure times) for wavelength calibration. Standard calibration frames were collected each night, including twilight sky spectra, bias frames, and quartz lamp flat-field spectra. On most nights we also observed telluric, RV, and spectrophotometric standard stars using the same instrumental setup.

We used the ccdproc routine in IRAF 3 to bias-subtract and flat-field the spectra, then optimally extracted each aperture with the apall function. Finally, we used the ecidentify routine in IRAF to identify comparison lamp emission lines and construct wavelength solutions. The ThAr solutions typically used $\sim 100$ lines and resulted in an RMS scatter of $\sim 300 \mathrm{~m} \mathrm{~s}^{-1}$ per line (approximately one thirtieth of a pixel).

After discarding orders severely contaminated by sky emission lines, CCD fringing, or cross-disperser order

\footnotetext{
3 IRAF is distributed by the National Optical Astronomy Observatories, which are operated by the Association of Universities for Research in Astronomy, Inc., under cooperative agreement with the National Science Foundation
}

overlap, as well as crowded or low-throughput orders, we found the useful wavelength coverage to be $\sim 4400-6200$ $\AA$. Since the data were taken without dividing the exposures to aid cosmic ray removal (so-called "splits"), we developed an IDL code to detect and record the locations of cosmic ray events in the extracted spectra. We made no attempt, however, to remove these or adjust the flux in these pixel locations but simply ignored compromised wavelength bins during the analysis that followed.

\section{ANALYSIS METHODS}

\subsection{Classifying the cool companions}

We estimated the spectral types of the cool companions by comparing the observed HET spectra to highresolution templates in the ELODIE 3.1 stellar spectral library (Prugniel \& Soubiran 2001). Spectral types F0M9 were considered in luminosity classes IV and V. Using our own IDL code, we compared the best individual spectrum for each target to the template spectra over the wavelength range 5075-5200 $\AA$, which includes important discriminators such as the $\mathrm{Mg} \mathrm{I} b$ triplet $(+\mathrm{MgH})$ and $\mathrm{Fe}$ lines. Before any comparisons were made, we degraded the resolution of the ELODIE templates $(\mathrm{R}=42,000)$ by convolving them with Gaussians to match the resolution of the HET/MRS observations $(\mathrm{R}=10,000)$. We constructed a library of possible "composite-spectrum" models, diluting each ELODIE template spectrum by a flat continuum (representing the contribution from the hot subdwarf) using a range of dilution factors (5-95\%). We then normalized the observed MRS spectra with high-order polynomials using the continuum function in IRAF and shifted each spectrum to its rest-frame.

For each object-template pair we subtracted the model from the object and computed the sum of squared residuals (SSR); the adopted best-fitting dilution is the one that minimizes the SSR. We repeated this process for all ELODIE templates and assigned a final spectral type for each cool companion target corresponding to the global SSR minimum. To test the accuracy of our fitting technique, we also classified several twilight-sky spectra and a dozen RV standards with known spectral types (drawn from the Astronomical Almanac and Nidever et al. 2002). Our results matched the known values reasonably well, falling within two subtypes of the published classification $80 \%$ of the time. The largest disagreement encountered in our consistency check was 4 subtypes.

The second column in Table 1 lists our classifications for the target stars, which range from $\mathrm{F} 4 \mathrm{~V}$ to $\mathrm{G} 8 \mathrm{~V}$. With $80 \%$ confidence, they are accurate to within 2 subtypes. They are also reasonably consistent with previously published values (e.g., Stark 2005). Although we report all companions as dwarfs, two of the targets, PG 0039+049 and PG 1317+123, are matched equally well with subgiant templates. Classifications could not be made for PG 1206+165 (companion lines too weak) or PG 1629+081 (resolved binary, companion not on fiber). In these cases, we adopt the spectral types reported by Stark (2005).

\subsection{Measuring the radial velocities}

We used IRAF routines to find RVs of the targets. Our primary strategy was to exploit the abundance of 

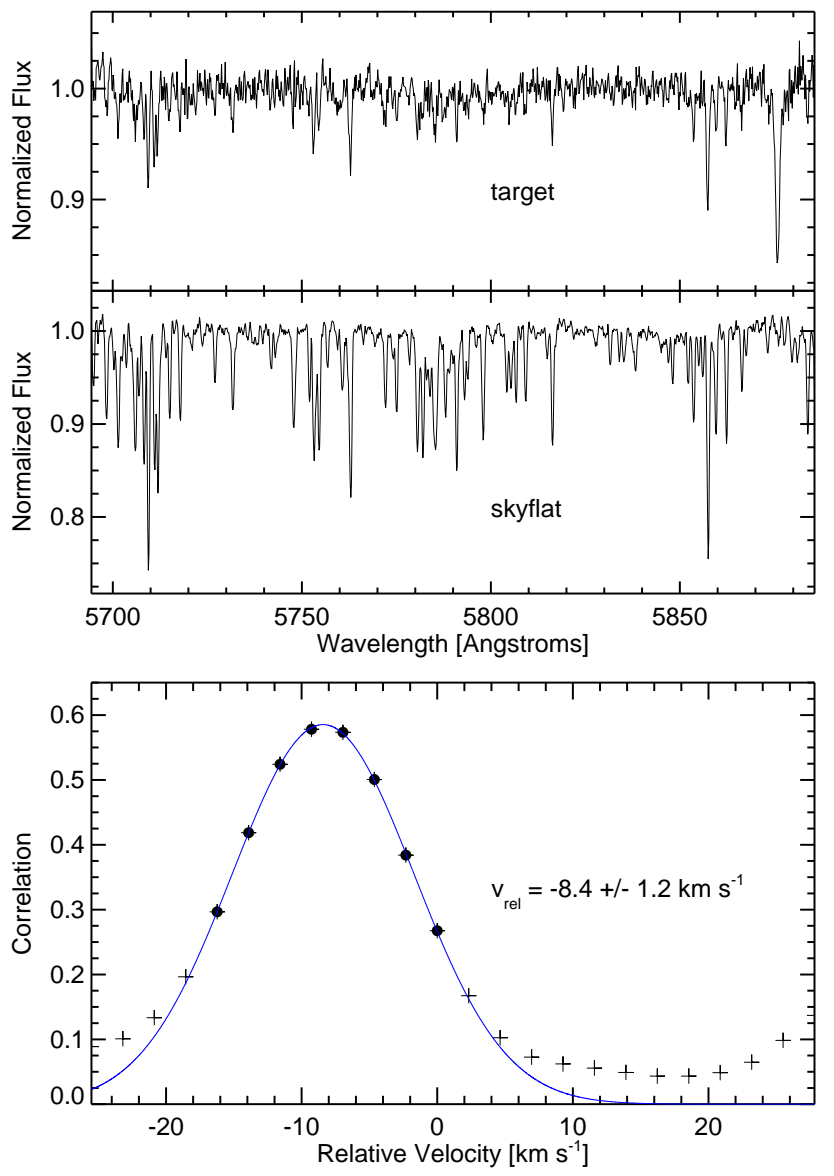

Fig. 1.- Example cross-correlation of a target-sky spectrum pair. A single order of one of the PG $1104+243$ spectra (top panel) is shown above the same order of a twilight-sky spectrum (middle panel). The He I $5876 \AA$ line from the sdB star stands out in the target spectrum amongst a plethora of features from the cool companion. The cross-correlation function (bottom panel) shows a reasonable match between the cool companion and solar features. The blue line shows the best-fitting Gaussian to the correlation values marked with solid points.

spectral features from the cool companion by crosscorrelating each spectrum against a twilight sky (i.e., solar) spectrum obtained on the same night. Before this was done, we created sampling region files for each spectrum that defined acceptable wavelength bins to use for the velocity fitting. In addition to ignoring bins compromised by cosmic rays and sky emission features, we also avoided spectral regions heavily influenced by absorption lines from the hot subdwarf component, namely the Balmer lines $(\mathrm{H} \beta, \mathrm{H} \gamma)$, He I lines $(4472,4713,5015$, $5876 \AA$ ) and the He II $4686 \AA$ line. Using only these data samples, we used the IRAF continuum task to fit the continuum and normalize it to unity. We then crosscorrelated the normalized spectra against the solar spectra, order-by-order, using fxcor and rvcorrect to determine heliocentric RVs.

Figure 1 1 shows an example target-sky pair and its cross correlation function. Most targets matched the solar spectrum with a correlation nearing 0.8 , although systems with companions deviating significantly from G2 V had lower values. Our fitting process resulted in $\sim 14 \mathrm{RV}$ measurements per target observation, depending on the exact number of echelle orders used. RV errors were computed from the standard deviation of these values about the mean. We find typical errors of $600-1100 \mathrm{~m} \mathrm{~s}^{-1}$.

In some cases, it was also possible to estimate the $\mathrm{sdB}$ star's RV from the available He I lines and, for the hotter stars, He II $4686 \AA$. We avoided using $\mathrm{H} \beta$ for this purpose since its profile spans almost an entire echelle order; also, its core is blended with the $\mathrm{H} \beta$ profile of the cool companion. For each of these targets, after selecting sampling regions around the sdB He lines, we cross-correlated each spectrum against a self-template, chosen to be the highest $\mathrm{S} / \mathrm{N}$ spectrum obtained for the target. This process only provides relative RV measurements with respect to this template; converting them to absolute velocities requires determining the heliocentric velocity of the template. Fitting simple Gaussians or other symmetric functions to the sdB lines cannot be used for this purpose since the He I lines are asymmetric and would skew the measured centroids. Instead, we cross-correlated the observed profiles against a model sdB spectrum with $T_{\text {eff }}$ $=30,000 \mathrm{~K}, \log g=5.6$, and $\log \mathrm{N}(\mathrm{He}) / \mathrm{N}(\mathrm{H})=-2$. We then adjusted the relative measurements appropriately. Naturally, the RV precision achieved from this process is not ideal, but it was sufficient to detect and measure $\mathrm{sdB}$ accelerations in many of the systems. RVs determined from He I lines may show a systematic offset, however, as some of the lines are sensitive to pressure shifts in the high-gravity atmosphere (especially the $5876 \AA$ $\mathrm{D}_{3}$ line). Additionally, the gravitational redshift difference between the cool companion and the compact hot subdwarf can give the appearance of a $\sim 2 \mathrm{~km} \mathrm{~s}^{-1}$ offset. These issues have been encountered before in studies of hot subdwarfs (e.g., Orosz et al. 1997) and also DB white dwarfs (e.g., Koester 1987), where the offset can approach tens of $\mathrm{km} \mathrm{s}^{-1}$. We will discuss their effects on our results object-by-object, as needed.

To investigate the zero-point accuracy of the MRS velocities, we cross-correlated all of the observed RV standards against twilight sky spectra (usually taken on the same night) and compared our results to the Nidever et al. (2002) values. A histogram of the RV differences is described reasonably well by a Gaussian distribution with a dispersion of $\sigma \approx 340 \mathrm{~m} \mathrm{~s}^{-1}$; its centroid is within $100 \mathrm{~m} \mathrm{~s}^{-1}$ of zero. We performed a similar test using all of the available twilight sky spectra, crosscorrelating them against the highest $\mathrm{S} / \mathrm{N}$ twilight sky spectrum. In this case, we find a scatter with $\sigma=250 \mathrm{~m}$ $\mathrm{s}^{-1}$. Our RV standard star scatter is roughly consistent with the twilight sky result, once the additional scatter of $\approx 150 \mathrm{~m} \mathrm{~s}^{-1}$ from the Nidever et al. (2002) measurements is added in quadrature.

\subsection{Fitting the orbital parameters}

We fitted each RV curve with a model of the form:

$$
V_{\mathrm{rad}}=\gamma+K[\cos (\theta+\omega)+e \cos (\omega)]
$$

where $\omega$ is the periastron angle, $K$ the RV semiamplitude, $\gamma$ the systemic velocity, and $e$ the eccentricity. The true anomaly $(\theta)$ is defined in terms of the period $(P)$, eccentricity, and time of periastron passage $\left(T_{0}\right)$ through the relation 


$$
\tan \left(\frac{\theta}{2}\right)=\sqrt{\frac{1+e}{1-e}} \tan \left(\frac{E}{2}\right)
$$

and Kepler's equation

$$
E-e \sin E=M=\frac{2 \pi}{P}\left(t-T_{0}\right)
$$

where $E$ is the eccentric anomaly and $M$ the mean anomaly. We used the IDL-based RVLIN package4 (Wright \& Howard 2009), which uses a LevenbergMarquardt algorithm for the non-linear parts of this optimization problem. Orbital parameter uncertainties were estimated using a bootstrapping technique driven by RVLIN (Wang et al. 2012). We determined the best solution with all parameters left free, and we also fitted the data assuming a circular orbit ( $e=0$ fixed), with two fewer degrees of freedom.

We also computed the floating-mean periodogram (e.g., Cumming et al. 1999) for each RV data set and investigated $\chi^{2}$ as a function of orbital period over the range $P=0.1-10,000 \mathrm{~d}$. We consider the orbital period "solved" if (i) the best-fitting orbit has a $\chi^{2}$ value at least 10 times smaller than the second-best alias and (ii) the uncertainty in $P$ for the best fit is less than $5 \%$. Once the first condition was met, we used a range of initial parameter guesses in RVLIN to be certain we converged on the correct solution, sometimes changing the inputs only slightly.

\subsection{Circular or eccentric?}

Determining the eccentricity of long-period sdB binaries is an astrophysical imperative. As already mentioned in $\$ 1$ if the main sequence companions are responsible for the formation of the $\mathrm{sdB}$ (and the sdB progenitor filled its Roche lobe), one would expect their orbits to have $e=0$ due to the circularizing nature of the RLOF and CE processes. If, however, the hot subdwarf formed from the merger of a binary originally part of a triple-star system, the cool companion currently observed would have had no part in the formation of the hot subdwarf, in which case the orbit could be non-circular. All of the longperiod systems studied to date have sufficient room to accommodate an inner binary, even after allowing for moderate "adiabatic expansion" of the orbit (Eggleton et al. 1989; Debes \& Sigurdsson 2002) resulting from mass loss in the $\mathrm{sdB}$ formation process.

In order to determine whether eccentric orbit fits are preferred for our targets, we performed an F-test similar to Lucy \& Sweeney (1971) using the statistic defined by

$$
\mathcal{F}=\frac{\left(\chi_{\text {circ }}^{2}-\chi_{\text {ecc }}^{2}\right)}{\left(D O F_{\text {ecc }}-D O F_{\text {circ }}\right)} \frac{D O F_{\text {ecc }}}{\chi_{\text {ecc }}^{2}}
$$

where $D O F_{\text {ecc }}$ and $D O F_{\text {circ }}$ are the degrees of freedom in the eccentric and circular orbit models, respectively, and $\chi_{\text {ecc }}^{2}$ and $\chi_{\text {circ }}^{2}$ the chi-squared values of their fits to the data. Under the assumption that $\mathcal{F}$ follows an $\mathrm{F}$ distribution, this statistic provides a quantitative means for deciding whether we can justify fitting the data with two additional degrees of freedom $(e, \omega)$ compared to our null hypothesis, a circular orbit solution. As $\mathcal{F}$ increases,

\footnotetext{
4 http://exoplanets.org/code/
}

so does the likelihood that one can discredit the null hypothesis. We reject a circular orbit solution if the probability of obtaining the observed $\mathcal{F}$ (the p-value) falls below our false-rejection probability, which we conservatively set to $10^{-4}$ due to the considerable phase gaps in the RV measurements. If the $\mathrm{p}$-value is above this limit, we do not rule out a circular-orbit solution using the current data.

\subsection{Computing the mass ratio}

For those systems for which we can measure the RVs of both components, we can calculate the mass ratio $q$ of the binary. It is straightforward to show that the instantaneous sdB and main-sequence (MS) companion RVs at time $t_{i}$ are related by

$$
V_{\mathrm{MS}}\left(t_{i}\right)=\gamma(1+q)-q V_{\mathrm{sdB}}\left(t_{i}\right)
$$

where $q \equiv K_{\mathrm{MS}} / K_{\mathrm{sdB}}=M_{\mathrm{sdB}} / M_{\mathrm{MS}}$, and $\gamma_{\mathrm{sdB}}=\gamma_{\mathrm{MS}}$ has been assumed. Thus, $q$ can be measured from the slope of a linear fit to a plot of $R V_{M S}$ versus $R V_{s d B}$. The resulting $q$ is unaffected by any systematic offsets between the component velocities, such as that caused by different gravitational redshifts. It is also independent of the inclination angle, which remains unknown for all systems studied (none show eclipses). We used the IDL fitexy 5 function, an orthogonal distance regression routine (procedure from Press et al. 1992), to determine $q$ from the best straight-line fit to the data, taking into account the errors from both radial velocity measurements.

\section{RESULTS}

All but two systems monitored in our program show some sort of orbital acceleration; most appear to have periods in excess of 100 days. Given such long periods and insufficient phase coverage, we cannot fully solve for the orbital parameters of the majority of targets using the 2005-2008 data alone. Table 2 summarizes our initial survey results, including the maximum (absolute) RV change observed for each target, the range of possible orbital periods, and the best-fitting orbital period taken from the model with the lowest $\chi^{2}$ in the periodogram (given in parentheses for the unsolved systems). Please note that the solutions for the unsolved systems are preliminary; they may change significantly with the addition of new data. The four binaries previously reported as resolved are likely triple-star systems since their observed accelerations are incompatible with the linear separation distances implied by their angular separations on the sky 6 . Two of these systems show RV variations of the main sequence star (PG 1040+234, PG 1718+519), while the other two show significant sdB motions (PG $1253+284$, PG 1629+081). Previous observations of PG $1253+284$ showing acceleration of the sdB but not the companion led Heber et al. (2002) to a similar triplestar system hypothesis for this system. We are currently conducting follow-up observations of unsolved systems with $\mathrm{HET} / \mathrm{HRS}$ and will report their orbital parameters in future publications, as they become available.

$\varnothing 12$ began a similar RV monitoring program with Mercator/HERMES in 2009, shortly after the HET observa-

\footnotetext{
5 http://idlastro.gsfc.nasa.gov/

6 Estimates of the minimum projected linear separation distances for the candidate triple systems range from 200 to $800 \mathrm{AU}$.
} 
TABLE 2

Preliminary Survey Results

\begin{tabular}{|c|c|c|c|c|}
\hline Target & $\begin{array}{l}\text { No. }^{a} \\
\text { Obs. }\end{array}$ & $\begin{array}{c}\mathrm{RV} \\
\text { variable? }\end{array}$ & $\begin{array}{l}\text { Period } \\
\text { [days] }\end{array}$ & $\begin{array}{l}\Delta R V_{\max } \\
{\left[\mathrm{km} \mathrm{s}^{-1}\right]}\end{array}$ \\
\hline \multicolumn{5}{|c|}{ Unsolved Systems } \\
\hline PG $0039+049$ & 7 & yes $^{b}$ & $150-300(220)$ & 11 \\
\hline PG $0110+262$ & 9 & $?$ & ? & - \\
\hline PB 8783 & 8 & $?$ & $?$ & - \\
\hline PHL 1079 & 5 & $\operatorname{yes}^{b}$ & $80-500(122)$ & 24 \\
\hline PG $0232+095$ & 6 & yes $^{b}$ & $<200(14)$ & 29 \\
\hline PG $1040+234$ & 9 & $\operatorname{yes}^{b}$ & $>500(700)$ & 12 \\
\hline PG $1206+165$ & 6 & yes $^{b}$ & ? & 15 \\
\hline PG $1253+284$ & 11 & yes $^{c}$ & $<220(161)$ & 93 \\
\hline PG $1449+653$ & 8 & yes $^{b}$ & $750-1000(859)$ & 15 \\
\hline PG $1629+081$ & 8 & yes $^{c}$ & $2-11(2.9)$ & 107 \\
\hline PG $1701+359$ & 14 & yes $^{b}$ & $630-830(725)$ & 9 \\
\hline PG $1718+519$ & 8 & yes $^{b}$ & $200-330(300)$ & 7 \\
\hline \multicolumn{5}{|c|}{ Solved Systems } \\
\hline PG $1104+243$ & 10 & yes $^{b, c}$ & 753.2 & 16 \\
\hline PG $1317+123$ & 11 & yes $^{b, c}$ & 1179 & 13 \\
\hline PG $1338+611$ & 11 & yes $^{b, c}$ & 937.5 & 16 \\
\hline
\end{tabular}

tions reported here ended. Three of their targets (PG $1104+243$, PG $1317+123$, PG 1338+611) overlap with the HET sample. By phasing together both observation sets, we are able to improve upon their original system constraints significantly. In the case of PG $1104+243$, we also include RVs obtained in 1995 with the GoldCam spectrograph on the $2.1 \mathrm{~m}$ telescope at Kitt Peak National Observatory (Orosz et al. 1997). We discuss all three systems and their updated orbital parameters below. Tables 3 and 4 present the heliocentric RVs of the cool companion and hot subdwarf, respectively, while Table 5 gives the fitted system parameters described in $\S 4.3$ along with their formal errors from the multi-parameter fits.

The systemic velocity $(\gamma)$ is subject to a possible zeropoint offset, so although we quote only the formal errors in the table, we refer the reader back to the discussion of our zero-point checks in 4.2 for a more complete understanding of their likely uncertainties. Due to the low quality of the hot subdwarf RVs, the orbital parameters shown $\left(P, K_{\mathrm{MS}}, \gamma, e, \omega, T_{0}\right)$ were derived from the cool companion measurements alone. The $K_{\mathrm{sdB}}$ values reported were calculated from $K_{\mathrm{MS}}$ and $q$, as described in $\S 4.5$; in all three cases, they are consistent with direct orbit fits to the hot subdwarf RVs if $P$ is taken from the cool companion orbital fit and the relation $\omega_{\mathrm{sdB}}=\omega_{\mathrm{MS}}+\pi$ is imposed. Finally, we detected no significant velocity offsets between telescopes, so we fixed their relative zeropoint offsets to zero during the final orbit fitting.

\section{1. $P G 1104+243$}

Previous classifications for the cool companion of $\mathrm{PG}$ $1104+243$ have ranged from F8 V (Stark 2005) to K3.5 V (Allard et al. 1994). Comparisons of the HET/MRS spectra to the ELODIE templates show a best-fitting spectral type near G2 V. The dilution of the companion spectrum is $D \equiv L_{\text {comp }} / L_{\text {total }} \approx 0.5$, where the luminosity ratio refers to the $5075-5200 \AA$ spectral region.
As noted by $\varnothing 12$, the hot subdwarf in PG $1104+243$ might be described best as an 'sdOB' star since its spectrum shows the He II $4686 \AA$ line in addition to strong $\mathrm{H}$ Balmer and He I features. Previous RV measurements have been published by Orosz et al. (1997), who found no significant variations over a three-day timespan. An orbital solution with $P=752 \pm 14 \mathrm{~d}$ was recently published by $\varnothing 12$. Combining our data with those of both $\varnothing 12$ and Orosz et al. (1997), we find an orbital period of $P=758.8 \pm 0.13 \mathrm{~d}$, in excellent agreement with the $\varnothing 12$ result. This value falls frustratingly close to two years, as clearly seen in the RV curve shown in Figure 2, By chance, current observing seasons align with quadrature phases; otherwise, all points would have been obtained near a conjunction, a less-than-ideal situation for measuring the mass ratio from spectroscopy. At the same time, information near conjunction is needed to improve constraints on the geometry of the orbit. Several more orbital cycles must elapse before complete phase coverage can be obtained. At this time, we have no reason to prefer an eccentric orbit over a circular one.

The presence of strong He lines from the sdB ( 5876 \& $4686 \AA$ ) permits us to measure accelerations for both components. As expected for stars in a binary, the sdB and MS velocity curves are 180 degrees out of phase. The sdB RV curve shows a systemic velocity consistent with that measured from the companion $(\gamma=-15.5 \pm 0.03 \mathrm{~km}$ $\left.\mathrm{s}^{-1}\right)$. From the relationship between $\mathrm{RV}_{\mathrm{sdB}}$ and $\mathrm{RV}_{\mathrm{MS}}$, as seen in Figure 3. we compute a mass ratio of $q=0.68$ \pm 0.08 . Table 5 summarizes the system parameters derived for PG $1104+243$. If we assume a companion mass of $1.0 \mathrm{M}_{\odot}$ and adopt a $10 \%$ error to include uncertainties in the spectral classification, we find $\mathrm{M}_{\mathrm{sdB}}=0.68 \pm$ $0.11 \mathrm{M}_{\odot}$, considerably larger than the 'canonical' value of $\sim 0.48 \mathrm{M}_{\odot}$, which corresponds to helium core ignition at the tip of the RGB. If we instead assume exactly the canonical mass for the hot subdwarf, we would compute a mass of $0.7 \pm 0.1 \mathrm{M}_{\odot}$ for the companion, which implies its spectral type might be significantly later than our result from the composite spectrum analysis, or that the companion is evolved.

\section{2. $P G 1317+123$ (Feige 80)}

Spectral classifications for the hot component in $\mathrm{PG}$ $1317+123$ have varied over years. It is probably an sdO star as it displays a strong He II $4686 \AA$ line and a relatively weak He I $4472 \AA$ line. Ulla \& Theill (1998) classify the cool companion as a G8 V, while $\varnothing 12$ report it to be a ' $G$ ' star. We find a spectral type near G1 V and dilution $D \approx 0.3$ from our comparisons with ELODIE models. $\varnothing 12$ reported an orbital period of $P=912 \pm$ 52 d. After phasing the HET data with theirs, we find a significantly longer value, $P=1179 \pm 12$ d. Figure 2 shows the resulting RV curve, while Table 5 summarizes all of the orbital parameters derived. Our results for the systemic velocity and MS RV amplitudes also disagree. As pointed out by $\varnothing 12$, the Mercator/HERMES measurements show much larger scatter than one would expect given the precision of their data. They briefly suggested $\gamma$ Doradus-type pulsations or star spots on the cool companion as potential culprits. However, Gtype classifications for the cool companion are inconsistent with those of $\gamma$ Dor stars, which are typically early $\mathrm{F}$ 
TABLE 3

Cool Companion Velocities for Solved Systems

\begin{tabular}{|c|c|c|c|c|c|c|c|c|}
\hline $\begin{array}{c}\text { HJD } \\
-2450000\end{array}$ & $\begin{array}{c}\mathrm{RV}_{\mathrm{MS}} \\
{\left[\mathrm{km} \mathrm{s}^{-1}\right]}\end{array}$ & Facility $^{a}$ & $\begin{array}{c}\text { HJD } \\
-2450000\end{array}$ & $\begin{array}{c}\mathrm{RV}_{\mathrm{MS}} \\
{\left[\mathrm{km} \mathrm{s}^{-1}\right]}\end{array}$ & Facility $^{a}$ & $\begin{array}{c}\text { HJD } \\
-2450000\end{array}$ & $\begin{array}{c}\mathrm{RV}_{\mathrm{MS}} \\
{\left[\mathrm{km} \mathrm{s}^{-1}\right]}\end{array}$ & Facility $^{a}$ \\
\hline \multicolumn{3}{|c|}{ PG $1104+243$} & \multicolumn{3}{|c|}{ PG $1317+123$} & \multicolumn{3}{|c|}{ PG $1338+611$} \\
\hline-215.23370 & $-16.80 \pm 4.70$ & $\mathrm{~K}$ & 3379.93771 & $45.66 \pm 1.09$ & $\mathrm{H}$ & 3379.95036 & $24.82 \pm 0.96$ & $\mathrm{H}$ \\
\hline-214.16710 & $-19.30 \pm 4.50$ & $\mathrm{~K}$ & 3392.92593 & $44.28 \pm 1.00$ & $\mathrm{H}$ & 3400.89553 & $25.62 \pm 1.21$ & $\mathrm{H}$ \\
\hline-213.24680 & $-17.30 \pm 4.70$ & $\mathrm{~K}$ & 3421.84076 & $44.04 \pm 0.68$ & $\mathrm{H}$ & 3415.89117 & $27.84 \pm 0.77$ & $\mathrm{H}$ \\
\hline-212.32400 & $-17.60 \pm 4.40$ & $\mathrm{~K}$ & 3446.76922 & $44.01 \pm 0.63$ & $\mathrm{H}$ & 3429.87462 & $28.35 \pm 0.57$ & $\mathrm{H}$ \\
\hline 3379.84221 & $-20.53 \pm 0.74$ & $\mathrm{H}$ & 3476.68120 & $42.92 \pm 0.92$ & $\mathrm{H}$ & 3476.70485 & $31.35 \pm 0.63$ & $\mathrm{H}$ \\
\hline 3392.80703 & $-20.20 \pm 0.72$ & $\mathrm{H}$ & 3488.65464 & $42.87 \pm 0.91$ & $\mathrm{H}$ & 3488.66280 & $32.69 \pm 0.96$ & $\mathrm{H}$ \\
\hline 3415.95642 & $-20.06 \pm 0.56$ & $\mathrm{H}$ & 3500.62543 & $42.13 \pm 0.70$ & $\mathrm{H}$ & 3502.64537 & $32.77 \pm 0.64$ & $\mathrm{H}$ \\
\hline 3429.73480 & $-19.63 \pm 0.41$ & $\mathrm{H}$ & 3724.01307 & $36.39 \pm 0.51$ & $\mathrm{H}$ & 3731.99294 & $40.23 \pm 0.78$ & $\mathrm{H}$ \\
\hline 3447.65376 & $-18.90 \pm 0.54$ & $\mathrm{H}$ & 3771.87931 & $35.42 \pm 0.78$ & $\mathrm{H}$ & 3732.97699 & $40.65 \pm 0.73$ & $\mathrm{H}$ \\
\hline 3462.62302 & $-19.61 \pm 0.40$ & $\mathrm{H}$ & 4453.00102 & $48.53 \pm 1.23$ & $\mathrm{H}$ & 4479.96424 & $35.36 \pm 0.78$ & $\mathrm{H}$ \\
\hline 3476.78809 & $-19.43 \pm 0.51$ & $\mathrm{H}$ & 4551.75800 & $45.21 \pm 0.90$ & $\mathrm{H}$ & 4540.80436 & $37.03 \pm 0.83$ & $\mathrm{H}$ \\
\hline 3498.74435 & $-19.12 \pm 0.46$ & $\mathrm{H}$ & 5030.42431 & $34.08 \pm 0.09$ & $\mathrm{M}$ & 5028.44884 & $27.83 \pm 0.02$ & $\mathrm{M}$ \\
\hline 3757.80663 & $-11.03 \pm 0.40$ & $\mathrm{H}$ & 5030.42431 & $34.08 \pm 0.09$ & $\mathrm{M}$ & 5028.47026 & $27.77 \pm 0.02$ & $\mathrm{M}$ \\
\hline 4479.82452 & $-10.97 \pm 0.78$ & $\mathrm{H}$ & 5030.45097 & $32.69 \pm 0.17$ & $\mathrm{M}$ & 5028.49174 & $27.77 \pm 0.03$ & $\mathrm{M}$ \\
\hline 5204.70741 & $-12.24 \pm 0.09$ & $\mathrm{M}$ & 5030.45097 & $32.69 \pm 0.17$ & $\mathrm{M}$ & 5341.57223 & $30.81 \pm 0.03$ & $\mathrm{M}$ \\
\hline 5204.72527 & $-12.37 \pm 0.04$ & $\mathrm{M}$ & 5294.54985 & $39.10 \pm 0.10$ & $\mathrm{M}$ & 5351.41840 & $31.07 \pm 0.05$ & $\mathrm{M}$ \\
\hline 5217.67376 & $-12.29 \pm 0.10$ & $\mathrm{M}$ & 5294.54985 & $39.10 \pm 0.10$ & $\mathrm{M}$ & 5371.41748 & $32.84 \pm 0.02$ & $\mathrm{M}$ \\
\hline 5217.69174 & $-12.27 \pm 0.10$ & $\mathrm{M}$ & 5353.40135 & $39.53 \pm 0.11$ & $\mathrm{M}$ & 5566.78096 & $40.00 \pm 0.04$ & $\mathrm{M}$ \\
\hline 5234.60506 & $-11.42 \pm 0.04$ & $\mathrm{M}$ & 5353.40135 & $39.53 \pm 0.11$ & $\mathrm{M}$ & 5616.64021 & $40.29 \pm 0.02$ & $\mathrm{M}$ \\
\hline 5234.61608 & $-12.09 \pm 0.11$ & $\mathrm{M}$ & 5373.41275 & $41.12 \pm 0.08$ & $\mathrm{M}$ & 5616.64021 & $40.29 \pm 0.02$ & $\mathrm{M}$ \\
\hline 5234.62710 & $-12.02 \pm 0.12$ & $\mathrm{M}$ & 5373.41275 & $41.12 \pm 0.08$ & $\mathrm{M}$ & 5622.70741 & $39.98 \pm 0.04$ & $\mathrm{M}$ \\
\hline 5264.54423 & $-11.15 \pm 0.07$ & $\mathrm{M}$ & 5566.74478 & $46.66 \pm 0.13$ & $\mathrm{M}$ & 5622.73169 & $39.96 \pm 0.03$ & $\mathrm{M}$ \\
\hline 5340.42821 & $-11.93 \pm 0.07$ & $\mathrm{M}$ & 5566.74478 & $46.66 \pm 0.13$ & $\mathrm{M}$ & 5656.60318 & $39.64 \pm 0.04$ & $\mathrm{M}$ \\
\hline 5351.37760 & $-11.91 \pm 0.08$ & $\mathrm{M}$ & 5616.61438 & $47.49 \pm 0.06$ & $\mathrm{M}$ & 5658.58702 & $39.98 \pm 0.08$ & $\mathrm{M}$ \\
\hline 5553.70059 & $-18.20 \pm 0.11$ & $\mathrm{M}$ & 5616.61438 & $47.49 \pm 0.06$ & $\mathrm{M}$ & 5660.61069 & $39.86 \pm 0.04$ & $\mathrm{M}$ \\
\hline 5569.69768 & $-18.78 \pm 0.09$ & $\mathrm{M}$ & 5616.61438 & $47.49 \pm 0.06$ & $\mathrm{M}$ & 5663.54111 & $39.90 \pm 0.03$ & $\mathrm{M}$ \\
\hline 5579.56553 & $-19.22 \pm 0.12$ & M & 5616.61438 & $47.49 \pm 0.06$ & $\mathrm{M}$ & 5666.53453 & $39.63 \pm 0.02$ & $\mathrm{M}$ \\
\hline 5579.58348 & $-18.93 \pm 0.09$ & $\mathrm{M}$ & 5640.61157 & $45.88 \pm 0.06$ & $\mathrm{M}$ & & & \\
\hline 5589.75559 & $-19.06 \pm 0.10$ & $\mathrm{M}$ & 5640.61157 & $45.88 \pm 0.06$ & $\mathrm{M}$ & & & \\
\hline 5611.64081 & $-19.73 \pm 0.07$ & M & 5653.60091 & $44.58 \pm 0.11$ & $\mathrm{M}$ & & & \\
\hline 5622.61268 & $-19.95 \pm 0.08$ & $\mathrm{M}$ & 5653.60091 & $44.58 \pm 0.11$ & $\mathrm{M}$ & & & \\
\hline 5639.58778 & $-19.57 \pm 0.07$ & $\mathrm{M}$ & 5661.63454 & $45.09 \pm 0.09$ & M & & & \\
\hline 5650.50737 & $-20.16 \pm 0.07$ & $\mathrm{M}$ & 5661.63454 & $45.09 \pm 0.09$ & $\mathrm{M}$ & & & \\
\hline 5652.55550 & $-19.99 \pm 0.05$ & $\mathrm{M}$ & 5722.45431 & $44.93 \pm 0.24$ & $\mathrm{M}$ & & & \\
\hline 5655.60537 & $-19.87 \pm 0.07$ & $\mathrm{M}$ & 5722.45431 & $44.93 \pm 0.24$ & $\mathrm{M}$ & & & \\
\hline 5658.47913 & $-20.10 \pm 0.06$ & M & & & & & & \\
\hline 5664.51354 & $-20.17 \pm 0.07$ & M & & & & & & \\
\hline 5666.43280 & $-20.41 \pm 0.05$ & $\mathrm{M}$ & & & & & & \\
\hline 5666.45153 & $-20.32 \pm 0.06$ & $\mathrm{M}$ & & & & & & \\
\hline 5672.53688 & $-20.30 \pm 0.11$ & $\mathrm{M}$ & & & & & & \\
\hline 5685.44968 & $-20.02 \pm 0.06$ & $\mathrm{M}$ & & & & & & \\
\hline 5718.44565 & $-19.50 \pm 0.06$ & $\mathrm{M}$ & & & & & & \\
\hline
\end{tabular}

or late A dwarfs. The other scenario, a bright spot on the companion's surface, seems more compatible with the observations. Assuming a solar-type dwarf and a star spot with a filling factor of a few percent (Saar \& Donahue 1997), the amplitude of the scatter $\left(1-2 \mathrm{~km} \mathrm{~s}^{-1}\right)$ would imply a rotational period around one week. As with PG 1104+243, we currently have no reason to prefer an eccentric-orbit solution. Even with additional data, constraining the eccentricity will be especially difficult until the rapid velocity variations are better characterized.

He I $5876 \AA$ and He II $4686 \AA$ features provided RV estimates for the hot subdwarf that are again 180 degrees out of phase with the cool companion. Figure 3 shows a strong linear relationship between $\mathrm{RV}_{\mathrm{sdO}}$ and $\mathrm{RV}_{\mathrm{MS}}$, whose slope gives $q=0.42 \pm 0.05$. A circular orbit fitted to the sdO RVs with the period and phase fixed to the values in Table 5 gives a consistent systemic velocity and a semi-amplitude in agreement with the expectation from combining $K_{\mathrm{MS}}$ and $q$. Assuming a companion mass from with our spectral classification, $M_{\mathrm{MS}}=1.03$ $\pm 0.11 \mathrm{M}_{\odot}$, gives $\mathrm{M}_{\mathrm{sdO}}=0.43 \pm 0.07 \mathrm{M}_{\odot}$.

\section{3. $P G 1338+061$ (Feige 87)}

Our analysis of PG 1338+061's spectrum reveals a G2G7V companion (best fit: G4V with $D \approx 0.2$ ), much earlier than previous classifications (K0 V, Stark 2005; K4.5 V, Allard et al. 1994). Two He I lines (5876 \& 4472 $\AA$ ) were used to measure the sdB RVs, which show the sdB and companion accelerating in anti-phase with one another. The last panel in Figure 2 presents the RV curve for PG 1338+061, combining the HET and Mercator observations. Unlike PG $1104+243$ and PG $1317+123$, we find significant evidence for an orbit with non-zero eccentricity. Figure 4 shows our best-fitting circular and eccentric fits to the cool companion's RV curve for compar- 
TABLE 4

Hot Subdwarf Velocities for Solved Systems from HeT/MRS

\begin{tabular}{|c|c|c|c|c|c|}
\hline $\begin{array}{c}\text { HJD } \\
-2450000\end{array}$ & $\begin{array}{c}\mathrm{RV}_{\mathrm{MS}} \\
{\left[\mathrm{km} \mathrm{s}^{-1}\right]}\end{array}$ & $\begin{array}{c}\text { HJD } \\
-2450000\end{array}$ & $\begin{array}{c}\mathrm{RV}_{\mathrm{MS}} \\
{\left[\mathrm{km} \mathrm{s}^{-1}\right]}\end{array}$ & $\begin{array}{c}\text { HJD } \\
-2450000\end{array}$ & $\begin{array}{c}\mathrm{RV}_{\mathrm{MS}} \\
{\left[\mathrm{km} \mathrm{s}^{-1}\right]}\end{array}$ \\
\hline \multicolumn{2}{|c|}{ PG $1104+243$} & \multicolumn{2}{|c|}{ PG $1317+123$} & \multicolumn{2}{|c|}{ PG $1338+611$} \\
\hline 3379.84221 & $-7.8 \pm 1.9$ & 3379.93771 & $28.8 \pm 1.3$ & 3379.95036 & $41.8 \pm 3.6$ \\
\hline 3392.80703 & $-8.0 \pm 1.6$ & 3392.92593 & $31.9 \pm 2.6$ & 3400.89553 & $43.3 \pm 3.3$ \\
\hline 3415.95642 & $-9.6 \pm 2.8$ & 3421.84076 & $31.4 \pm 1.0$ & 3415.89117 & $39.1 \pm 2.9$ \\
\hline 3429.73480 & $-11.1 \pm 2.3$ & 3446.76922 & $32.2 \pm 2.2$ & 3429.87462 & $38.3 \pm 3.6$ \\
\hline 3447.65376 & $-11.3 \pm 1.1$ & 3476.68120 & $31.8 \pm 1.0$ & 3476.70485 & $32.5 \pm 2.4$ \\
\hline 3462.62302 & $-11.4 \pm 2.0$ & 3488.65464 & $34.6 \pm 3.0$ & 3488.66280 & $31.4 \pm 3.5$ \\
\hline 3476.78809 & $-12.0 \pm 2.3$ & 3500.62543 & $37.6 \pm 2.9$ & 3502.64537 & $33.1 \pm 4.4$ \\
\hline 3498.74435 & $-11.8 \pm 2.4$ & 3724.01307 & $50.1 \pm 1.8$ & 3731.99294 & $18.4 \pm 3.6$ \\
\hline 3757.88245 & $-22.5 \pm 1.5$ & 3771.87931 & $51.3 \pm 5.4$ & 3732.97699 & $16.0 \pm 2.1$ \\
\hline \multirow[t]{2}{*}{4479.87074} & $-23.6 \pm 2.3$ & 4453.00102 & $24.2 \pm 8.3$ & 4479.96424 & $28.3 \pm 3.9$ \\
\hline & & 4551.75800 & $31.2 \pm 3.0$ & 4540.80436 & $20.1 \pm 6.2$ \\
\hline
\end{tabular}

TABLE 5

Orbital Parameters for the Solved Systems

\begin{tabular}{|c|c|c|c|c|c|c|c|}
\hline $\begin{array}{l}\text { Target } \\
\text { Name }\end{array}$ & $\begin{array}{c}P \\
{[\text { days] }}\end{array}$ & $\begin{array}{c}K_{\mathrm{MS}} \\
{\left[\mathrm{km} \mathrm{s}^{-1}\right]}\end{array}$ & $\begin{array}{c}K_{\mathrm{sdB}^{a}} \\
{\left[\mathrm{~km} \mathrm{~s}^{-1}\right]}\end{array}$ & $\begin{array}{c} \\
{\left[\mathrm{km} \mathrm{s}^{-1}\right]}\end{array}$ & $\bar{e}$ & $\begin{array}{c}\omega \\
{[\mathrm{deg}]}\end{array}$ & $\begin{array}{c}T_{0}{ }^{c} \\
\text { [HJD-2450000] }\end{array}$ \\
\hline PG $1104+243$ & $753.2 \pm 0.8$ & $4.43 \pm 0.06$ & $6.5 \pm 0.8$ & $-15.68 \pm 0.05$ & $0.0^{b}$ & - & $4534.5 \pm 3.3$ \\
\hline PG $1317+123$ & $1179 \pm 12$ & $6.2 \pm 0.2$ & $15.5 \pm 1.7$ & $+40.3 \pm 0.2$ & $0.0^{b}$ & - & $4453 \pm 15$ \\
\hline PG $1338+611$ & $937.5 \pm 1.1$ & $8.8 \pm 0.15$ & $15.2 \pm 1.8$ & $+32.58 \pm 0.07$ & $0.15 \pm 0.02$ & $209 \pm 6$ & $4685.5 \pm 3.0$ \\
\hline
\end{tabular}

ison. Computation of the $\mathrm{F}$-test statistic gives $\mathcal{F}=12.6$ with a corresponding $\mathrm{p}$-value of $10^{-7}$, well below our criterion for claiming non-zero eccentricity. We therefore reject the circular-orbit hypothesis and report $e=0.15$ \pm 0.02 for this system. The best-fitting period in this case is $P=937 \pm 10 \mathrm{~d}$, in slight disagreement with $\varnothing 12$, who assumed a circular-orbit solution and found $P=$ $915 \pm 16 \mathrm{~d}$. In 6.2 we discuss the implications of nonzero eccentricity on formation scenarios of long-period sdB systems.

The relationship between $R V_{\mathrm{sdB}}$ and $R V_{\mathrm{MS}}$ (Figure 3) is fitted well with $q=0.58 \pm 0.07$. Combining this value with $K_{\text {MS }}$ predicts a velocity semi-amplitude consistent with the best-fit to the subdwarf RV curve (with period and phase fixed). The difference in the systemic velocities is $\gamma_{\mathrm{sdB}}-\gamma_{\mathrm{MS}}=-2.1 \pm 1.0 \mathrm{~km} \mathrm{~s}^{-1}$. Assuming a mass of $M_{\mathrm{MS}}=0.97 \pm 0.1 M_{\odot}$ for the companion, we calculate $M_{\mathrm{sdB}}=0.56 \pm 0.09 M_{\odot}$ for the hot subdwarf, consistent with the canonical value.

6. THE PERIOD DISTRIBUTION OF HOT SUBDWARF BINARIES REVISITED

Recent orbital solutions for long-period binaries have drastically changed the empirical period distribution of hot subdwarf binaries, compared with the situation a few years ago. We present in Figure 5] an updated period histogram for all systems with published orbital parameters. Most of the short-period systems can be found in Geier et al. (2011) (see Table A.1 and references therein), Copperwheat et al. (2011), and
Morales-Rueda et al. (2003). For the long-period binaries, which include PG 1018-047 (Deca et al. 2012), results from $\varnothing 12$, and all targets in our survey with reasonably well-constrained periods (see Table 2), we include estimates of the uncertainties in the orbital periods. Given the difficulties associated with monitoring the long-period systems, several strong observational biases are undoubtedly at play in the diagram; premature conclusions should not be drawn.

\subsection{The problem posed by long orbital periods}

One clear trend appears to emerge from the empirical distribution of orbital periods: closer binaries tend to have late-type MS or WD companions while the longperiod systems host $\mathrm{F} / \mathrm{G} / \mathrm{K}$ dwarfs. This apparent dichotomy has been noted before (e.g., Green 2008). Although the number of long-period binaries known is still relatively small, their addition allows us to begin testing the predictions of BPS models in both the short- and long-period regimes for the first time.

The Han et al. (2002, 2003) models reproduce the short-period end of the distribution quite well but predict that the $\mathrm{sdB}+\mathrm{MS}(\mathrm{G} / \mathrm{K})$ binaries should have periods shorter than $\sim 100 \mathrm{~d}$, the outcome of CE evolution using the " $\alpha$-formalism", in which orbital energy (and/or thermal energy stored in the envelope) is tapped to expel the envelope. Current observations suggest that the energy-based $\alpha$-formalism for CE evolution in its sim- 

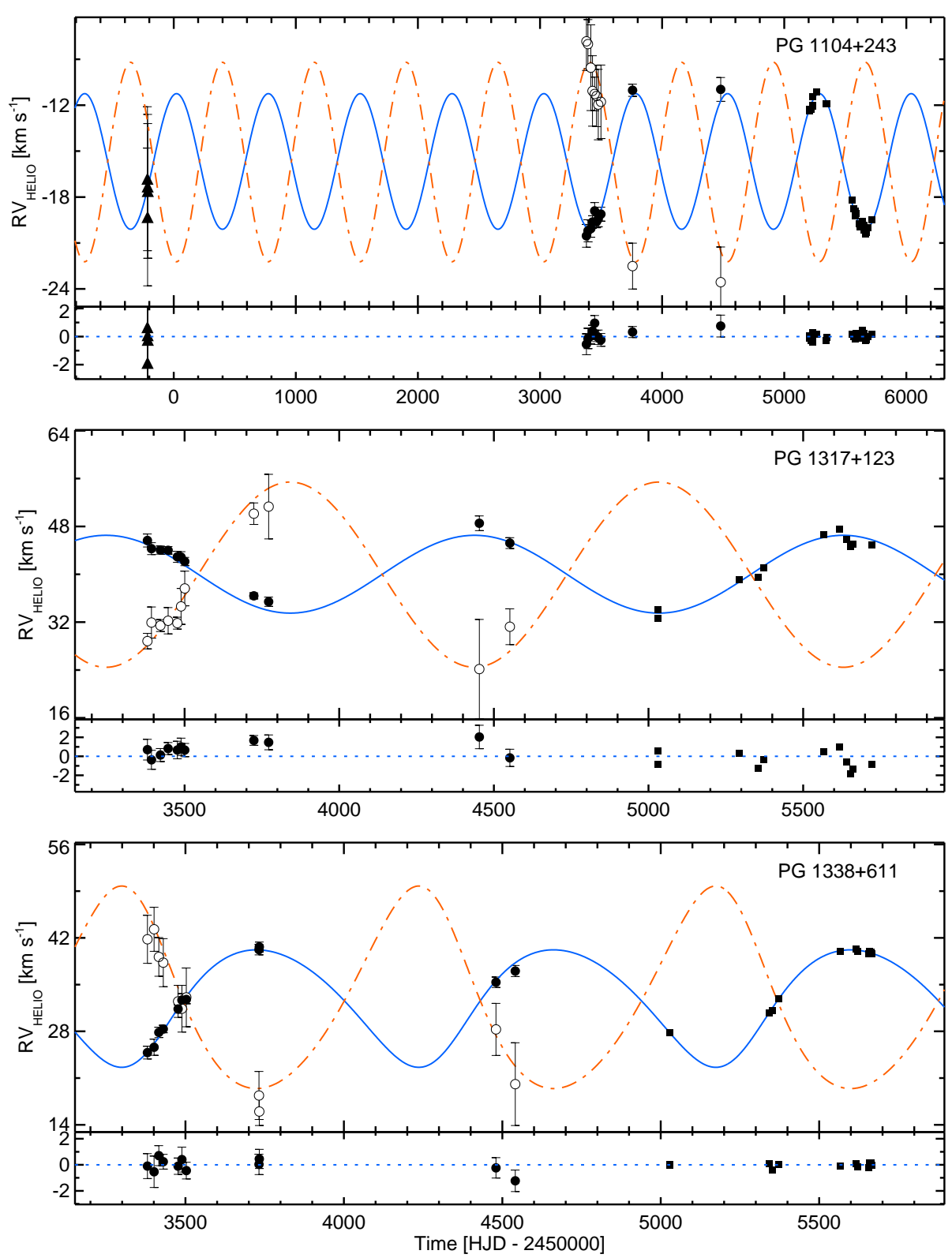

FIG. 2. - Heliocentric RV curves for the three targets overlapping with the study of $\varnothing 12$ : PG 1104+243 (top), PG 1317+123 (middle), and PG 1338+611 (bottom). Cool companion measurements from HET/MRS, Ø12, and Orosz et al. (1997) are shown with filled circles, squares, and triangles, respectively. Open circles represent sdB velocities measured from He lines in the HET/MRS spectra. The bestfitting orbital solutions for the sdB and cool companion are denoted with dot-dashed (orange) and solid (blue) lines, respectively. Residuals from this fit are shown in the lower portion of each panel for the cool companion measurements. Error bars on the Mercator/HERMES points are much smaller than the symbol sizes; we do not plot them here.

plest forms does not account for the long-period sdB binaries. We note that Han et al. (2002, 2003) deliberately excluded the GK-effect binaries when choosing their favored BPS models, focusing on the short-period M dwarf and WD systems (the only systems with measured periods available at the time). Thus, it is not surprising their model predictions are inconsistent with the current period distribution of $\mathrm{sdB}+\mathrm{F} / \mathrm{G} / \mathrm{KV}$ binaries. Clausen et al. (2012) encountered a similar problem when modeling the sdB population using the $\alpha-$ formalism. This failing is not limited to the sdB binaries and has been discussed in other contexts by Webbink (2008), who points out the need in some cases for (1) additional energy sources to augment or substitute for some of the energy extracted from the orbit in expelling the envelope, or (2) a reduction in the binding energy of the envelope (possibly through more extensive mass loss prior to the CE phase), in order that orbital shrinkage not be so drastic.

An alternative scenario proposed by Nelemans et al. (2000, 2001) appears, at first glance, to be more successful. In this model, the long-period systems are the product of $\mathrm{CE}$ evolution, with a prescribed fractional angular momentum loss that is proportional to the fractional mass lost when the envelope is ejected. The current distribution of long-period systems overlaps surpris- 

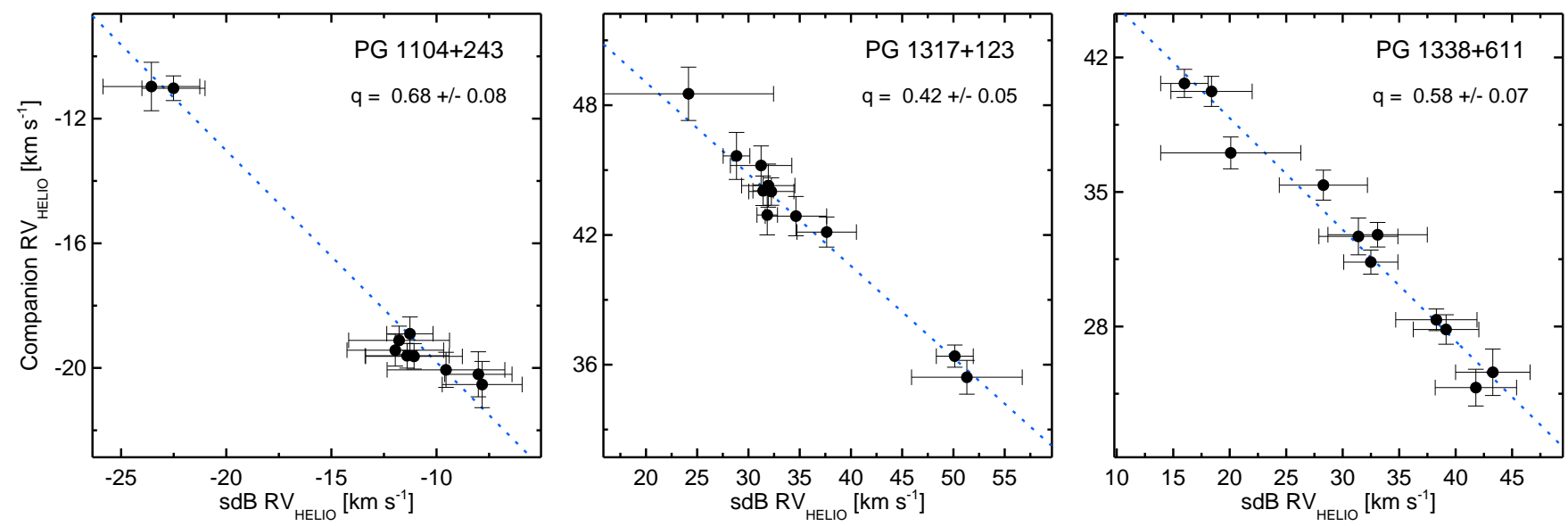

FIG. 3.- HET/MRS orbital velocities of the cool companion and sdB plotted against one another for PG 1104+243, PG 1317+123, and PG 1338+611. The dotted lines show the best-fitting linear functions to the data; the absolute values of their slopes are the mass ratios. Improved constraints on the mass ratios from HERMES measurements of the sdB velocities will be presented by Vos et al. (2012, in prep).

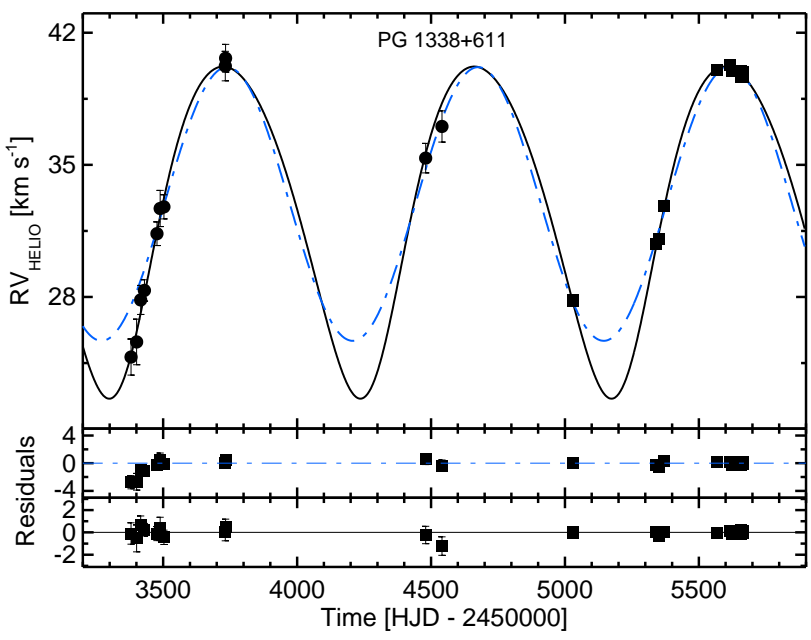

FIG. 4.- Heliocentric radial velocities for PG $1338+611$ with the best-fitting circular (blue dotted line) and eccentric (solid black line) orbits, and their residuals (bottom panels). Velocities from HET/MRS and $\varnothing 12$ are shown with circles and squares, respectively. The data are not consistent with a circular solution; the best-fitting eccentric model has $e=0.15 \pm 0.02$.

ingly well with the densest region of the predicted population when the angular momentum parameter $\gamma=1.5$ (Figure 2 of Nelemans 2010). Current observations hint at a dearth of binaries in the range $P \approx 30-110 \mathrm{~d}$, and in this context we note that a period histogram drawn 'by eye' from the sdB+MS population (with companion masses $\sim 1 M_{\odot}$ ) predicted by Nelemans (2010) shows a local minimum in the number of binaries with periods from $\approx 30$ to $90 \mathrm{~d}$. It would be well not to read too much into this apparent agreement, since the model is advertised as tentative, is not well-matched to the PG sample in terms of its flux limit, and the transformation of sdB bolometric luminosities to $V$ magnitudes is not described. In addition, the empirical diagram should be more thoroughly populated and the observational biases accounted for, before the significance of the gap is assessed.

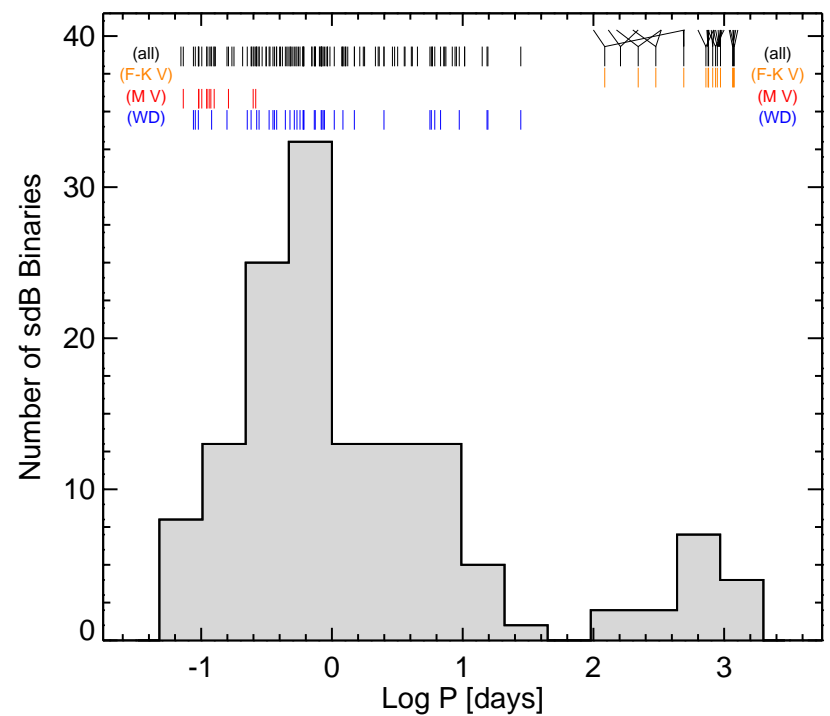

Fig. 5.- Histogram of the orbital periods of 140 hot subdwarf binaries with known orbital parameters. The black vertical lines at the top indicate the individual periods of all systems. For the long-period binaries with poor fits, they represent the best-fitting orbit in the periodogram; two diagonal lines emanating from the tops of these symbols mark the range of possible orbital periods. In the rows of vertical lines below the first, we re-mark systems with $\mathrm{F}-\mathrm{K}$ dwarf (second row, orange marks), $\mathrm{M}$ dwarf (third row, red marks), and white dwarf (fourth row, blue marks) companions. Those with uncertain companion classifications or none at all are not shown. The long-period systems exclusively have companions of spectral type $\sim \mathrm{K} 4$ or earlier. Although the statistics are poor, observations currently show a gap centered near $P=70 \mathrm{~d}$.

\subsection{Eccentricity as a clue to the long periods}

It is interesting to consider the question of eccentricity of the observed wide orbits. PG $1338+061$ (this paper) and possibly PG 1018-047 (Deca et al. 2012) have significant eccentricities. We also note that eccentric orbits have not been excluded for PG $1104+243$ and PG $1317+123$ based on the present data. If wide sdB binaries typically prove to have eccentric orbits, this would suggest one of three possibilities. Perhaps (1) these systems are or were hierarchical triple systems in which the sdB star was made in the inner binary, possibly by merger (see, e.g., Clausen \& Wade 2011, who describe a "hydro- 
gen merger channel" that leads to sdB's with canonical masses). We note that in PG $1338+061$, the minimum periastron distance of $2 \mathrm{AU}$ in the current orbit gives ample room to have once accommodated an inner system. If however the systems have always been binaries, then either (2) circularization was not achieved because the sdB progenitor never filled its Roche lobe or (3) eccentricity was pumped into the system via one of several possible mechanisms.

In the first case, the current long-period eccentric orbits are not directly comparable to the predictions of BPS models since the observed cool companions did not participate directly in the formation of the hot subwarfs. They nevertheless provide some dynamical constraints on "binary" formation scenarios, since they limit the size of the inner orbit in the hierarchical triple. Studies of the $\mathrm{sdB}$ rotational velocities in these systems might shed light on whether a merger included two $\mathrm{He}$-core WDs (fast rotation expected: Gourgouliatos \& Jeffery 2006; although if there is a giant stage between merger and the $\mathrm{sdB}$ phase the rotation may be slowed, see Zhang \& Jeffery 2012) or a He WD and a MS star (slow rotation, owing to angular momentum shedding near the tip of the RGB; Clausen \& Wade 2011).

In the case these systems have always been binaries, we suppose that a strong, tidally-enhanced wind along the lines suggested by Tout \& Eggleton (1988) can occasionally result in the complete loss of the envelope, just as the core of the sdB progenitor reaches the critical mass (or near it, see D'Cruz et al. 1996) to ignite He burning, without ever filling its Roche lobe. Some expansion of the orbit would occur owing to the wind mass loss, and the orbital eccentricity could in large part be preserved. If this timing coincidence does not occur, the extra mass loss can at least reduce the envelope binding energy and, after a CE episode, leave a (circular) orbit at longer period than would otherwise be attained, or even reduce the mass ratio to the point where RLOF mass transfer proceeds stably and a CE phase is avoided altogether, as in Tout \& Eggleton (1988).

Finally, we consider briefly the case that these systems are in fact binaries in which the sdB progenitor once filled its Roche lobe. Several mechanisms exist that could work to increase the orbital eccentricity, even as mass is being lost or transferred, thereby preventing the binaries from circularizing. Most notably, these include pumping from a circumbinary disk (e.g. Dermine et al. 2012 and references therein) and asymmetric/phase-dependent mass loss (Bonačić Marinović et al. 2008; Soker 2000), both of which have been invoked to explain the large eccentricities observed in some post-AGB systems having undergone strong tidal interactions. Long-period sdB binaries are post-RGB systems, and as such, their sdB progenitors had significantly different luminosities, radii, envelope structures, and mass-loss rates than AGB stars. Eccentricity-pumping mechanisms operating on RGB and AGB systems will neither act on the same timescales, nor will they have the same ability to compete with the effects of tides. We regard these scenarios, which have primarily been linked to post-AGB binaries, as uncertain in the case of hot subdwarf binaries; further discussion of them is beyond the scope of this paper. For completeness, we also recognize the action of a distant third body (the Kozai mechanism, Kozai 1962), which we regard as the 'ultimate epicycle', as another possible avenue for increasing the eccentricity.

\section{CONCLUDING REMARKS}

We have described an ongoing program to determine the orbital parameters of hot subdwarfs in binaries with $\mathrm{F}-\mathrm{K}$ type main sequence companions. Preliminary results show most of our targets have orbital periods on the order of years (rather than days), in agreement with the recent study by $\varnothing 12$ and the findings of Green et al. (2001) from more than a decade ago. Precise orbital solutions cannot be provided for most of our systems at this time, owing to insufficient phase coverage. Nonetheless, the preliminary findings imply that the assumptions inherent in some subdwarf formation scenarios should be revised, which probably requires both re-tuning the parameters used in BPS models and accounting for at least some of the long-period systems as former hierarchical triples.

For the three systems also observed by $\varnothing 12$, we calculated precise orbital parameters from nearly seven years of RV measurements. Mass ratios were derived from the orbital RVs and are consistent with our spectral classifications for the cool companion and canonical-mass hot subdwarfs for two of the three systems. In the case of the third, PG $1104+243$, the derived subdwarf mass exceeds the range classically expected for sdBs that evolved through the stable RLOF channel, implying the subdwarf either formed from a WD merger (resulting in a largerthan-canonical mass), or the companion has a mass different from what we infer from its spectral type.

A long-term goal for observers is to determine the joint distribution of orbital periods and eccentricities in longperiod sdB binary systems. At this time, we cannot rule out eccentric orbit solutions for PG $1104+243$ or PG $1317+132$. Determining the eccentricity of PG $1317+123$ will prove especially difficult due to the presence of rapid $R V$ variations, which might originate from star spots on the cool companion. We find a statistically significant eccentricity of $e=0.15 \pm 0.02$ for PG $1338+061$ and reject the circular-orbit hypothesis in this case. The longperiod binary PG 1018-047 also shows evidence in favor of a non-circular orbit (Deca et al. 2012), although this result needs confirmation. Such departures from $e=0$, taken at face value, challenge the prevailing notion that strong binary interactions, involving the filling of the sdB progenitor's Roche lobe, are always involved in the formation of hot subdwarf stars. If each of these binaries was (or is) a hierarchical triple system, then the cool companion that we observe probably did not participate directly in the formation of the hot subdwarf, in which case its orbit would not have been circularized. The hot subdwarf in this picture could have formed from the merger of two He-core WDs (Han et al. 2002, 2003) or a He-core WD and a MS star (Clausen \& Wade 2011). Measurements of the rotational velocities of sdBs in long-period systems might shed light on their formation by identifying the type of merger. On the other hand, if the system has always been a binary, a strong, tidally-enhanced wind (Tout \& Eggleton 1988) could have assisted the sdB progenitor in ejecting its envelope on the RGB before it expanded enough to fill its Roche lobe, thereby preserving some level of eccentricity in the binary. Even in the case the Roche lobe was filled previously, non- 
steady mass loss, the action of a distant third companion, or interactions with a circumbinary disc could have potentially added eccentricity to the orbit, although these scenarios seem less likely.

In light of the updated period histogram for sdB binaries (Fig. 5), we find it imperative that observers continue to study both short- and long-period systems with all possible types of companions, to arrive at a clear understanding of the formation channels for hot subdwarf stars. Thus it is important to give attention to issues of completeness and bias in our knowledge of binary orbits (or otherwise) for sdB stars. An important first step is to establish a more complete catalogue or finding list, as for example in the effort described by Girven et al. (2012), who search for photometrically composite objects and use decomposition of the spectral energy distribution to identify binaries containing hot subdwarfs. We note that one of the selection criteria of Girven et al. (2012) is that the binary is unresolved; this by itself does not indicate whether the binary is close enough to be tidally interacting, so follow-up studies are needed. Eventually the goal is to derive full orbital elements for an unbiased sample of any interacting binaries that are found in these surveys.
Meanwhile, work continues to characterize the orbits of sdB binaries that are already known. We are currently conducting a follow-up program with HET/HRS to determine the orbital periods, velocities, and eccentricities of the unsolved targets in our survey.

This material is based upon work supported by the National Science Foundation under Grant No. AST0908642. The Hobby-Eberly Telescope (HET) is a joint project of the University of Texas at Austin, the Pennsylvania State University, Stanford University, LudwigMaximilians-Universität München, and Georg-AugustUniversität Göttingen. The HET is named in honor of its principal benefactors, William P. Hobby and Robert E. Eberly. This work is also based on observations made with the Mercator Telescope, operated on the island of La Palma by the Flemish Community, at the Spanish Observatorio del Roque de los Muchachos of the Instituto de Astrofísica de Canarias. Finally, we thank an anonymous referee for comments and suggestions that helped to improve and balance discussions in the manuscript.

Facilities: HET (MRS), Mercator1.2m (HERMES), KPNO:2.1m (GoldCam)

\section{REFERENCES}

Allard, F., Wesemael, F., Fontaine, G., Bergeron, P., \& Lamontagne, R. 1994, AJ, 107, 1565

Berger, J., \& Fringant, A.-M. 1984, A\&AS, 58, 565

Bonačić Marinović, A. A., Glebbeek, E., \& Pols, O. R. 2008, A\&A, 480, 797

Clausen, D., \& Wade, R. A. 2011, ApJ, 733, L42

Clausen, D., Wade, R. A., Kopparapu, R. K., \& O'Shaughnessy, R. 2012, ApJ, 746, 186

Copperwheat, C. M., Morales-Rueda, L., Marsh, T. R., Maxted, P. F. L., \& Heber, U. 2011, MNRAS, 415, 1381

Cumming, A., Marcy, G. W., \& Butler, R. P. 1999, ApJ, 526, 890

D'Cruz, N. L., Dorman, B., Rood, R. T., \& O'Connell, R. W. 1996, ApJ, 466, 359

Debes, J. H., \& Sigurdsson, S. 2002, ApJ, 572, 556

Deca, J., Marsh, T. R., Østensen, R. H., et al. 2012, MNRAS, 421,2798

Dermine, T., Izzard, R. G., Jorissen, A., \& Van Winckel, H. 2012, A\&A, in press (arXiv:1203.6471)

Eggleton, P. P., Tout, C. A., \& Bailyn, C. D. 1989, ApJ, 345, 489

Geier, S., Hirsch, H., Tillich, A., et al. 2011, A\&A, 530, A28

Girven, J., Steeghs, D., Heber, U., et al. 2012, MNRAS, 425, 1013

Gourgouliatos, K. N., \& Jeffery, C. S. 2006, MNRAS, 371, 1381

Green, E. M., 2008, in ASP Conf. Ser., 392, Hot Subdwarf Stars and Related Objects, ed. U. Heber, C. S. Jeffery, \& R. Napiwotzki, 75

Green, E. M., Liebert, J., \& Saffer, R. A. 2001, in ASP Conf. Ser., Vol. 226, 12th European Workshop on White Dwarfs, ed. J. L. Provencal, H. L. Shipman, J. MacDonald, \& S. Goodchild (San Francisco, CA: ASP), 192

Green, R. F., Schmidt, M., \& Liebert, J. 1986, ApJS, 61, 305

Han, Z., Podsiadlowski, P., Maxted, P. F. L., \& Marsh, T. R. 2003, MNRAS, 341, 669

Han, Z., Podsiadlowski, P., Maxted, P. F. L., Marsh, T. R., \& Ivanova, N. 2002, MNRAS, 336, 449

Haro, G., \& Luyten, W. J. 1962, Boletin de los Observatorios Tonantzintla y Tacubaya, 3,37

Heber, U. 1986, A\&A, 155, 33

Heber, U., Moehler, S., Napiwotzki, R., Thejll, P., \& Green, E. M. 2002, A\&A, 383, 938

Kilkenny, D. 1984, MNRAS, 211, 969

Koen, C., Kilkenny, D., O'Donoghue, D., van Wyk, F., \& Stobie, R. S. 1997, MNRAS, 285, 645

Koester, D. 1987, ApJ, 322, 852

Kozai, Y. 1962, AJ, 67, 591

Lucy, L. B., \& Sweeney, M. A. 1971, AJ, 76, 544
Maxted, P. F. L., Heber, U., Marsh, T. R., \& North, R. C. 2001 MNRAS, 326, 1391

Mengel, J. G., Norris, J., \& Gross, P. G. 1976, ApJ, 204, 488

Morales-Rueda, L., Maxted, P. F. L., Marsh, T. R., North, R. C., \& Heber, U. 2003, MNRAS, 338, 752

Nelemans, G. 2010, Ap\&SS, 329, 25

Nelemans, G., Verbunt, F., Yungelson, L. R., \& Portegies Zwart, S. F. 2000, A\&A, 360, 1011

Nelemans, G., Yungelson, L. R., Portegies Zwart, S. F., \& Verbunt, F. 2001, A\&A, 365, 491

Nidever, D. L., Marcy, G. W., Butler, R. P., Fischer, D. A., \& Vogt, S. S. 2002, ApJS, 141, 503

Orosz, J., Wade, R. A., \& Harlow, J. J. B. 1997, AJ, 114, 317

Østensen, R., Heber, U., \& Maxted, P. 2005, in ASP Conf. Ser., Vol. 334, 14th European Workshop on White Dwarfs, ed. D. Koester \& S. Moehler (San Francisco, CA: ASP), 435

Østensen, R. H., \& Van Winckel, H. 2012, in ASP Conf. Ser., Vol. 452, Fifth Meeting on Hot Subdwarf Stars and Related Objects, ed. D. Kilkenny, C. S. Jeffery, \& C. Koen (San Francisco, CA: ASP), 163

Østensen, R. H. 2012, in ASP Conf. Ser., Vol. 452, Fifth Meeting on Hot Subdwarf Stars and Related Objects, ed. D. Kilkenny, C. S. Jeffery, \& C. Koen (San Francisco, CA: ASP), 233

Press, W. H., Teukolsky, S. A., Vetterling, W. T., \& Flannery, B. P. 1992, Numerical recipes in FORTRAN. The art of scientific computing (Cambridge, UK: Cambridge Univ. Press)

Prugniel, P., \& Soubiran, C. 2001, A\&A, 369, 1048

Ramsey, L. W., Adams, M. T., Barnes, T. G., et al. 1998, in SPIE Conf. Ser., Vol. 3352, Advanced Technology Optical/IR Telescopes VI, ed. L. M. Stepp, 34-42

Raskin, G., van Winckel, H., Hensberge, H., et al. 2011, A\&A, 526, A69

Saar, S. H., \& Donahue, R. A. 1997, ApJ, 485, 319

Soker, N. 2000, A\&A, 357, 557

Stark, M. A. 2005, PhD thesis, The Pennsylvania State University Tout, C. A., \& Eggleton, P. P. 1988, ApJ, 334, 357

Ulla, A., \& Thejll, P. 1998, A\&AS, 132, 1

Wang, S. X., Wright, J. T., Cochran, W. D., et al. 2012, ApJ, submitted

Webbink, R. F. 2008, in Short-Period Binary Stars: Observations, Analyses, and Results, ed. E. F. Milone, D. A. Leahy, \& D. W. Hobill (Berlin: Springer Verlag), 233

Wright, J. T., \& Howard, A. W. 2009, ApJS, 182, 205

Zhang, X., \& Jeffery, C. S. 2012, MNRAS, 419, 452 